\title{
UNA PROPUESTA DE SOLUCIÓN AL PROBLEMA DE LA INTERFERENCIA ENTRE REDES WIFI POR SOLAPAMIENTO DE CANALES
}

\section{A PROPOSED SOLUTION TO THE PROBLEM OF INTERFERENCE BETWEEN WIRELESS NETWORKS BY OVERLAPPING CHANNELS}

\author{
Roberto Carlos Guevara C. \\ Facultad de Ciencias Básicas e Ingeniería \\ Corporación Universitaria Remington \\ Medellín, Colombia \\ roberto.guevara@remington.edu.co \\ Édgar Serna M. \\ Facultad de Ciencias Básicas e Ingeniería \\ Corporación Universitaria Remington \\ Medellín, Colombia \\ edgar.serna@remington.edu.co \\ Fecha de recepción: 13 Abril 2013 \\ Fecha de aprobación: 10 Diciembre 2013
}

\section{RESUMEN}

La amplia utilización de redes WiFi en los diversos escenarios sociales está generando una especie de saturación de canales, que a su vez ocasiona interferencia entre estas redes. Esta situación genera solapamiento de canales, lo que hace que las tasas de trasferencia se reduzcan. En este artículo se detalla esta situación, y se estructura y aplica un experimento para encontrarle solución al problema. También se presentan los resultados tabulados y graficados utilizando la herramienta de software libre inSSider.

Palabras clave: Interferencia, WiFi, solapamiento de canales, redes.

ABSTRACT

The widespread use of WiFi networks in different social settings is generating a sort of channel saturation, at the same time it causes interference among these networks. This situation generates channels overlap, making the transfer rates are reduced. This article 
details this situation, and is structure and applies an experiment to find a solution to the problem. It also presents the results tabulated and graphed using the free inSSIDer software.

Keywords: Interference, WiFi, overlapping channels, networks.

\section{INTRODUCCIÓN}

En escenarios como residencias, campus universitarios, empresas y sitios públicos es común el uso de redes WiFi, por lo que es posible que al instalar una nueva red en un área determinada su cobertura se expanda a zonas donde existen otras, lo que ocasiona interferencia entre ellas. La cercanía geográfica entre estas redes se traduce en bajas velocidades de transferencia, incluso si existen pocos computadores conectados.

En este artículo se explica la razón de estos inconvenientes y se describe una cuantificación experimental de la tasa de transferencia de archivos en redes WiFi cuando comparten un mismo canal; además, se hacen algunas recomendaciones acerca de cómo mejorar la comunicación y se plantea un procedimiento de planificación y selección de canales de comunicación para evitar esas interferencias.

En un aspecto más amplio los resultados de este trabajo se pueden utilizar en procesos industriales en lo relacionado con el monitoreo o control inalámbrico utilizando tecnologías Zigbee que estén en la misma zona geográfica de las redes WiFi. Tecnologías como Zigbee y WiFi trabajan en la banda de $2.4 \mathrm{Ghz}$, por lo que es latente la posi- bilidad de interferencia, pero conocer cómo cuantificarla y evitarla hace parte de una investigación más amplia orientada a reconocer la producida por la tecnología WiFi y por las redes industriales Zigbee y otros dispositivos inalámbricos que trabajen en la frecuencia ISM $2.4 \mathrm{Ghz}$, que son bandas reservadas internacionalmente para uso no comercial.

\section{ANTECEDENTES CONCEPTUALES}

Las redes inalámbricas 802.11 básicamente son inseguras [2] y pueden ser interferidas por una serie de elementos que funcionan en la frecuencia de $2.4 \mathrm{Ghz}$, como teléfonos inalámbricos, microondas, dispositivos bluetooth y dispositivos Zigbee entre otros [1-2]. Esa interferencia puede afectar la velocidad de transmisión nominal esperada para la red, la cual es de 54Mbps [2].

Esta interferencia la pueden causar factores como la modulación, el encapsulamiento de protocolos, la sintonización fina de la tarjeta de red y el router inalámbrico, lo mismo que los protocolos de encriptación utilizados y la distancia al router o al Access Point. Las redes en modo infraestructura se configuran usando un enrutador inalámbrico o Access Point [2] con un esquema como el que se muestra en la Figura 1. 


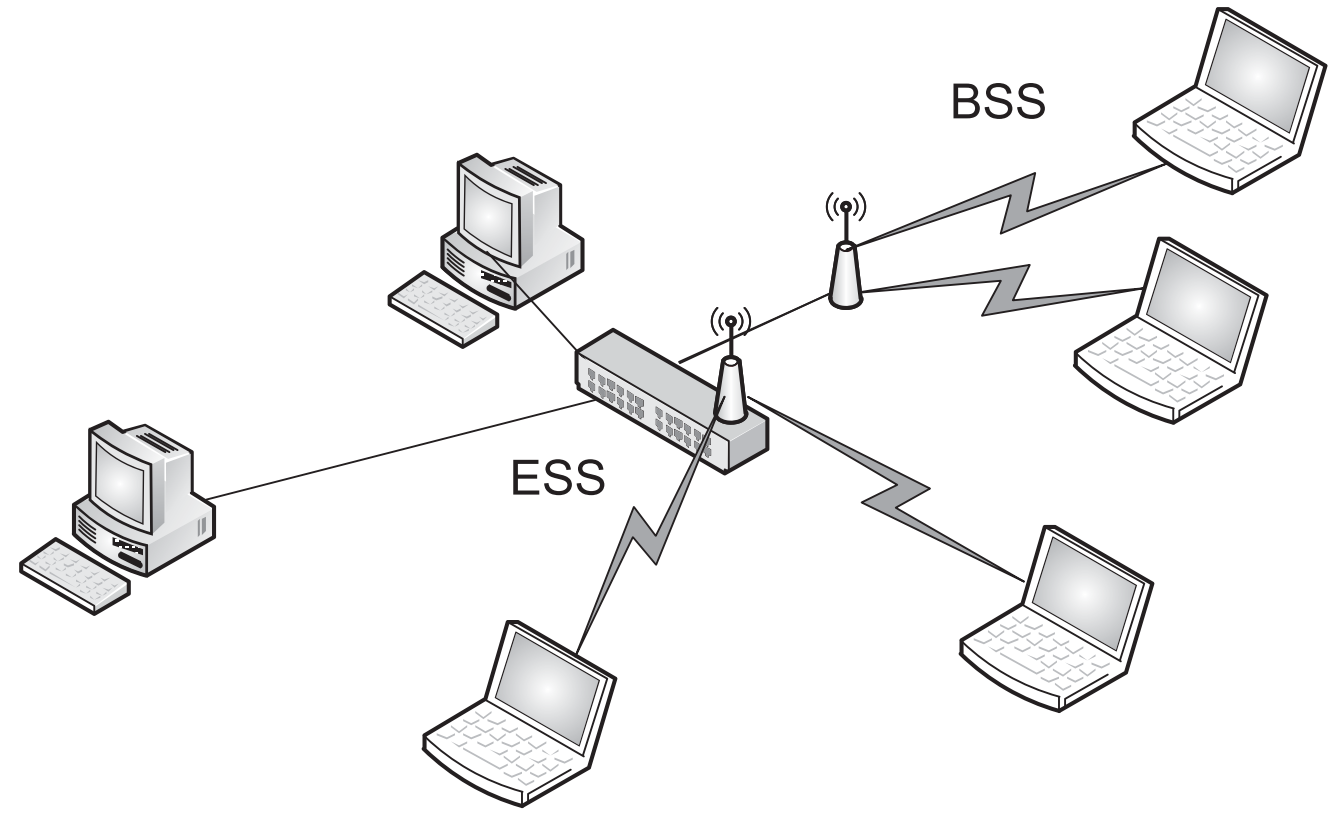

Figura 1. Modo infraestructura con Access Point

La configuración del punto de acceso y las estaciones ubicadas dentro del área de cobertura se llama Conjunto de Servicio Básico (BSS por sus siglas en inglés). En el modo infraestructura cada una de las redes WiFi tiene un identificador llamado BSSID de 48bits, que corresponde a la MAC del Access Point [2]. Se puede vincular varios BSS con una conexión de Sistema de Distribución (SD), conformando de esta manera un Conjunto de Servicio Extendido (ESS por sus siglas en inglés), el cual emplea generalmente un router inalámbrico y cada ESS y BSS debe estar ubicado en canales diferentes para evitar interferencias [2].

\subsection{CANALES EMPLEADOS EN LAS REDES WIFI}

La comunicación WiFi se establece a través de 14 canales y cada uno ocupa $22 \mathrm{MHz}$ de ancho de banda [2-3], como se muestra en la Tabla 1.

Tabla 1. Canales IEEE $802.11 \mathrm{~b} / \mathrm{g}$ Wifi

\begin{tabular}{|c|c|c|}
\hline Banda & Frecuencia & Canal \\
\hline $2.4 \mathrm{GHz}$ & $2412.0 \mathrm{MHz}$ & 1 \\
\hline $2.4 \mathrm{GHz}$ & $2417.0 \mathrm{MHz}$ & 2 \\
\hline $2.4 \mathrm{GHz}$ & $2422.0 \mathrm{MHz}$ & 3 \\
\hline $2.4 \mathrm{GHz}$ & $2427.0 \mathrm{MHz}$ & 4 \\
\hline $2.4 \mathrm{GHz}$ & $2432.0 \mathrm{MHz}$ & 5 \\
\hline $2.4 \mathrm{GHz}$ & $2437.0 \mathrm{MHz}$ & 6 \\
\hline $2.4 \mathrm{GHz}$ & $2442.0 \mathrm{MHz}$ & 7 \\
\hline $2.4 \mathrm{GHz}$ & $2447.0 \mathrm{MHz}$ & 8 \\
\hline $2.4 \mathrm{GHz}$ & $2452.0 \mathrm{MHz}$ & 9 \\
\hline $2.4 \mathrm{GHz}$ & $2457.0 \mathrm{MHz}$ & 10 \\
\hline $2.4 \mathrm{GHz}$ & $2462.0 \mathrm{MHz}$ & 11 \\
\hline $2.4 \mathrm{GHz}$ & $2467.0 \mathrm{MHz}$ & 12 \\
\hline $2.4 \mathrm{GHz}$ & $2472.0 \mathrm{MHz}$ & 13 \\
\hline $2.4 \mathrm{GHz}$ & $2484.0 \mathrm{MHz}$ & 14 \\
\hline
\end{tabular}


El estándar IEEE $802.11 \mathrm{~b} / \mathrm{g}$ sólo permite disposición de canales y el espaciado entre tres canales no interferentes espaciados a ellos y se resalta los canales no interferi$3 \mathrm{MHz}$ [2-4]. En la Figura 2 se muestra la bles.

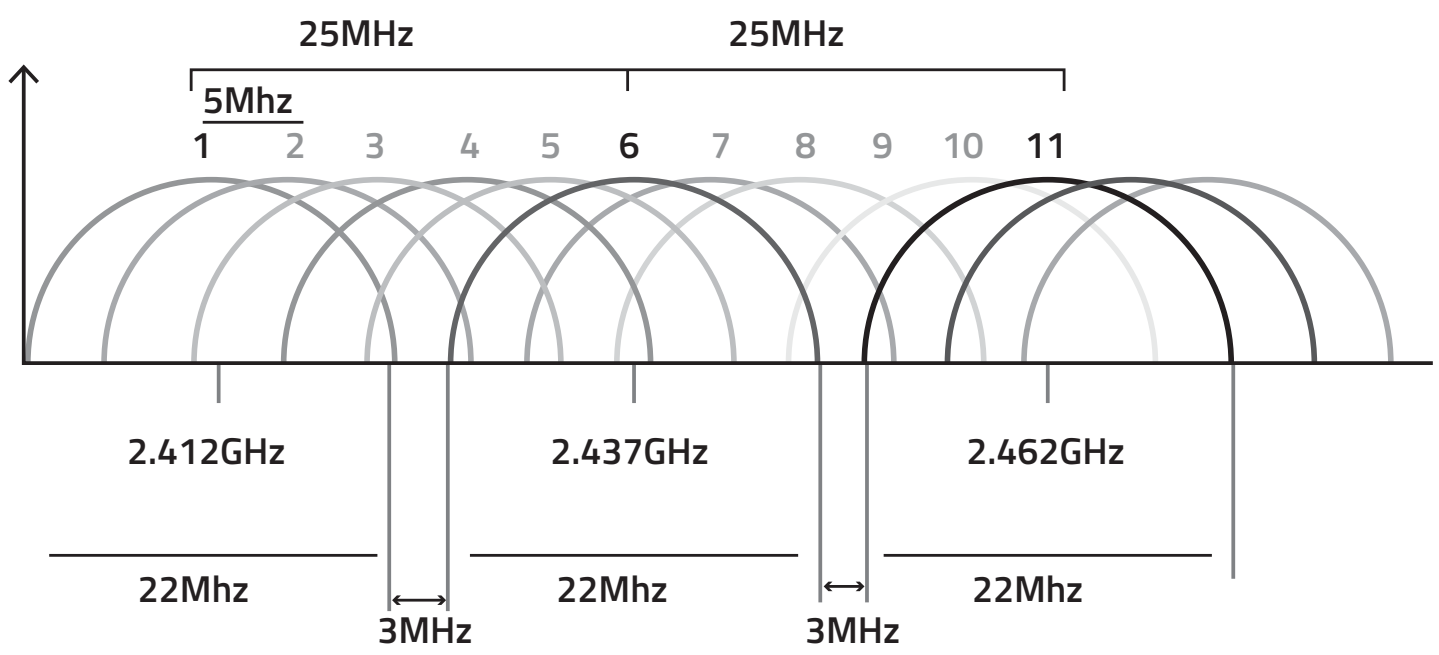

Figura 2. Canales No interferibles y anchos de banda

Utilizando el software InSSider [5] para analizar si los canales están solapados o no, se puede observar la asignación usada por los ESS y los BSS existentes y geográficamente cercanos.

\section{PROCEDIMIENTO DE ESCOGENCIA DE CANAL}

Al instalar una red WiFi se debe asegurar que no existe otro ESS trabajando en el mismo canal, y para lograrlo se utilizan analizadores de espectro, como se detalla en [5]. A continuación se describe un procedimiento para realizar la instalación de una red WiFi libre de interferencias, el cual se ilustra en la Figura 3.

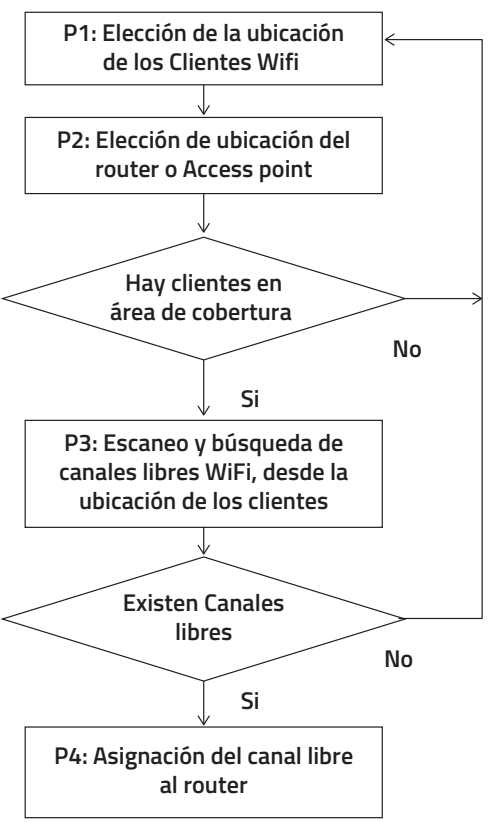

Figura 3. Pasos para instalación de una red WiFi 
Sin embargo, es posible que no se pueda acceder en la zona a un canal no interferible, porque pueden existir redes WiFi que estén ocupando los canales 1,6 y 11 . Incluso, pueden existir otras redes que utilicen canales que se solapen parcialmente, en este caso, se debe buscar uno donde el impacto de ese solapamiento sea mínimo.

\section{MATERIALES}

Para cuantificar experimentalmente la disminución de la tasa de transferencia de archivos en redes WiFi, que comparten un mismo canal, se realizó un montaje con los siguientes elementos:

- Software. El software empleado fue inSSider [5], un aplicativo de uso libre, el cual permite: Ver los ESSID de las redes inalámbricas que se encuentren en la zona, muestra gráficamente los canales usados, el solapamiento de las señales y la intensidad de trasmisión. Además, realiza la función de analizador de espectro utilizando la tarjeta de red inalámbrica.

- Hardware. Las pruebas se realizaron en un computador HP TouchSmart TX2 2010, con tarjeta Wireless LAN $802.11 \mathrm{a} / \mathrm{b} / \mathrm{g} / \mathrm{n}$ y tecnología Bluetooth. Otras especificaciones técnicas son:

- Procesador AMD Turion ${ }^{\mathrm{TM}}$ X2 RM-77 Dual-Core
- Memoria de 3072MB DDR2 a $800 \mathrm{MHz}$

- Disco Duro de 320GB SATA a 7200RPM

El router usado fue un NetGear WGT624, la Internet/WAN fue de 10/100 Mbps (autosensing) Ethernet, RJ-45 y la LAN: fue de 4 ports 10/100 Mbps (auto-sensing) Ethernet, RJ-45.

La Wireless utilizada fue una Network Speeds: 1, 2, 5.5, 6, 9, 11, 12, 18, 24,36, 48, \& $54 \mathrm{Mbps}$ (auto-rate capable) $108 \mathrm{Mbps}$ (Static and Dynamic), con Modulation Type OFDM with BPSK, QPSK, 16QAM, 64QAM, DBPSK, DQPSK, CCK, una Frequency Band de $2.4 \mathrm{GHz}$, con Standards Capability $802.11 \mathrm{~g}$ and $802.11 \mathrm{~b}$ y una Antenna $2 \mathrm{dBi}$ attached.

\subsection{ESCENARIO DE PRUEBAS}

Para las pruebas se utilizó un escenario real con variables no controladas, el cual es característico en diversos sitios residenciales donde existen varias redes WiFi, cada una con su propio ESSID y que convergen en un misma área geográfica, por lo que se interfieren entre sí.

Luego, se instaló una red de tipo infraestructura entre el computador y el router, y se conectó por cable un PC que comparte el archivo a transferir, tal como se ilustra en la 
Figura 4. La red de prueba se bautizó como ESSID RCGCalume, y se configuró el router usando el canal 11 interferido y otro no interferido, el canal 1.

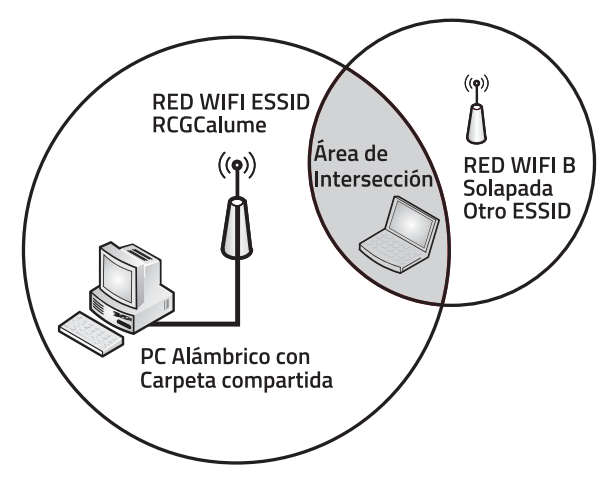

Figura 4. Infraestructura empleada

\subsection{ARCHIVO TRANSMITIDO}

Se realizó la transmision de un archivo tipo avi debido a su tamaño, además porque tiene un radio de compresion alto que no permite compresión durante la transmision.

\section{TOMA DE MUESTRAS}

El valor en $\mathrm{dBm}$ en un punto con potencia $\mathrm{P}$ se calcula por la ecuación (1).

$$
d B m=10 \log \frac{P}{1 m W}
$$

Si se requiere realizar operaciones más complejas sobre los $\mathrm{dBm}$, por ejemplo sacar un promedio, es necesario tener en cuenta que éstos se deben transformar a potencia, sacar el promedio y luego transformar el resultado nuevamente a $\mathrm{dBm}$, utilizando la ecuación (2).

$$
d B m_{\text {promedio }}=10 x \log \left(\frac{\sum_{\mathrm{i}=1}^{n} P_{n}}{n m W}\right)
$$

inSSIDer [5] utiliza las ecuaciones (1) y (2) para calcular y promediar los resultados obtenidos y los entrega gráficamente, como se muestra en la Figura 5.

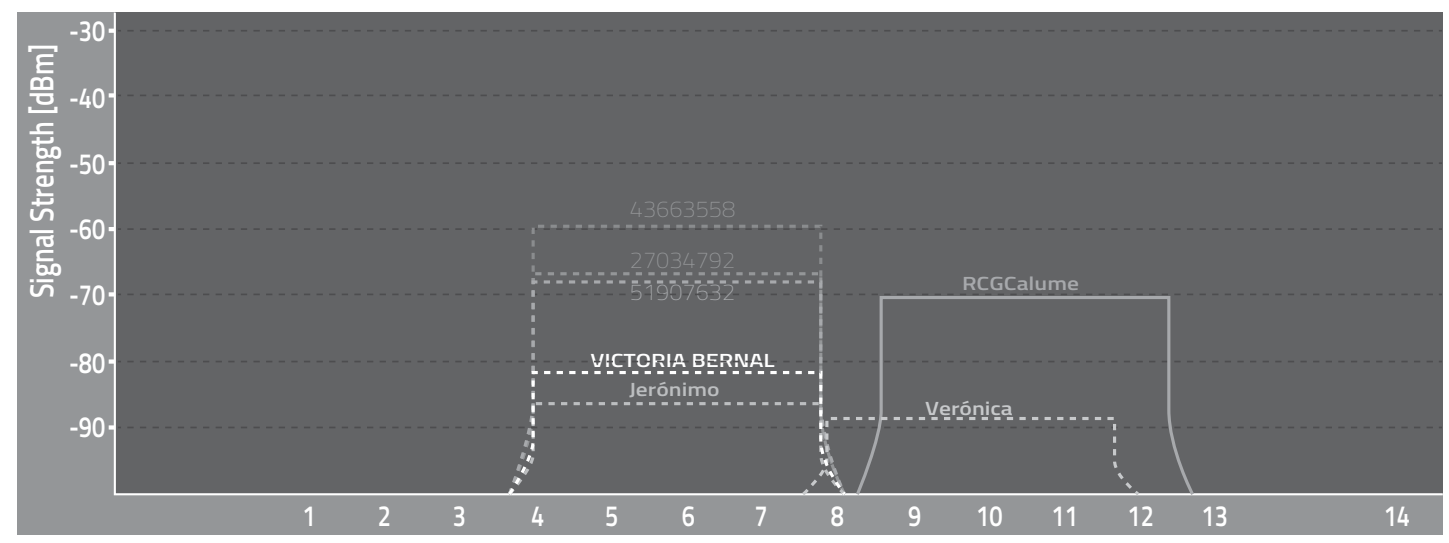

Figura 5. Configuración de la red de control RCGCalume en canal 11 
Para todas la transmisiones se aseguró una potencia del $100 \%$ en el computador con tarjeta Wifi; el envío del archivo de prueba se realizó 10 veces a través del canal 11, el cual presentaba interferencia con otras redes WiFi; luego se envió otras 10 veces a través del canal 1, libre de interferencias, y finalmente, el promedio se contrastó con la tasa de tranferencia para cada caso. La situación de distribución de las redes cercanas y los canales que usan se escanearon con inSSIDer.
La Figura 5 muestra que la red RCGCalume (en azul) está ubicada en el canal 11, y que se sola con la red Verónica, está ubicada en el canal 10. En esta situación la tasa de transferencia promedio fue de $384 \mathrm{~KB} / \mathrm{Se}-$ gundo. Además, se observa que el canal 1 está libre, por lo que se ubica el router en él y se envía nuevamente el mismo archivo; luego se promedia la velocidad de trasferencia. Posteriormente, se hace nuevamente un escaneo con el software inSSIDer de la nueva situación de la distribución, el cual se muestra en la Figura 6.

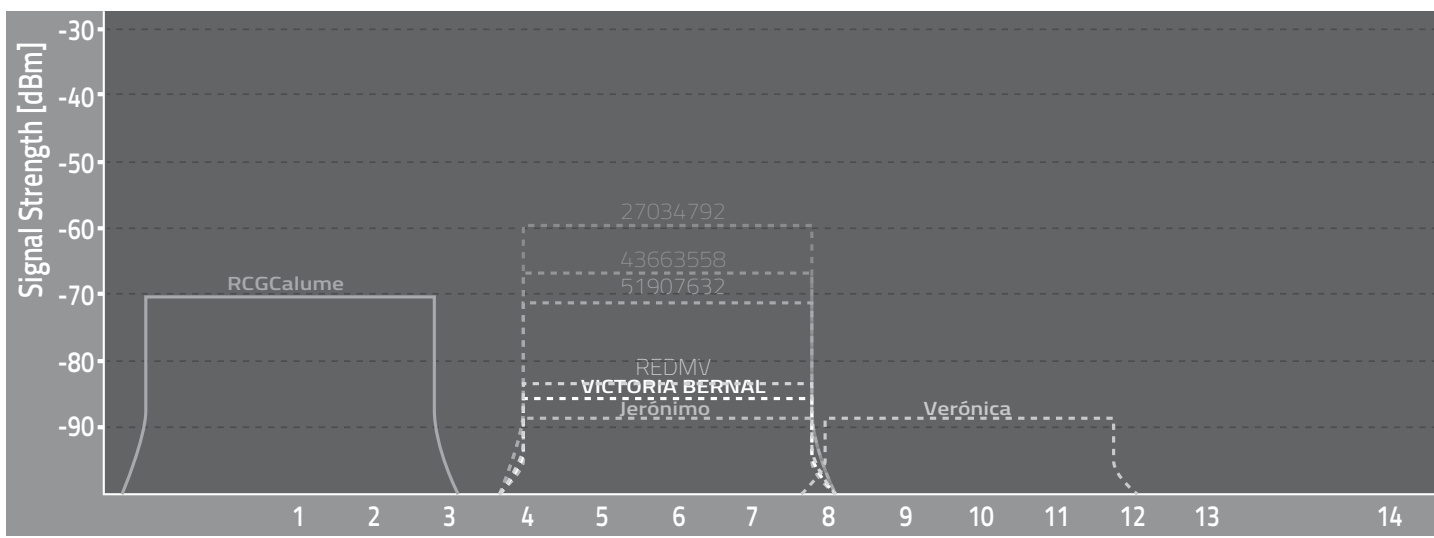

Figura 6. Distribución en los canales de las redes cercanas luego del cambio

La Figura 6 muestra que la red RCGCalume ahora está ubicada en el canal 1, donde no se solapa ni es interferida por otras redes; en esta situación la tasa de transferencia promedio de los 10 envíos fue de 1.93M (B// Segundo).

\section{RESULTADOS}

Aunque se use el método propuesto es posible que no se pueda acceder en la zona a un canal no interferible, debido a que pueden existir redes WiFi que ocupen los canales 1, 6 y 11 no interferibles. En la Tabla 
2 se muestra un listado de los canales no interferibles y sus solapamientos con otros canales.

Tabla 2. Canales No interferibles y posibles interferencias

\begin{tabular}{|c|l|}
\hline Canal no interferible & Posibles interefencias con \\
\hline 1 & $2,3,4$ y 5 \\
\hline 6 & $2,3,4,5,7,8,9$ y 10 \\
\hline 11 & $7,8,9,10,12,13$ y 14 \\
\hline
\end{tabular}

La trasferencia del archivo en el canal 11 con interferencia de la red Verónica mostró que el promedio de la velocidad de envío es $348 \mathrm{~KB} /$ segundo. La unidad de transferencia se da en Mbps y se realiza el cambio de unidades expresado en la ecuación (4).

$$
348 \text { KBps } * 8=2784 \text { Kbps }
$$

$$
\frac{2784 \mathrm{Kbps}}{1024}=2,71875 \mathrm{Mbps}
$$

Al enviar el archivo cuando no existe interferencia, es decir por el canal 1, se observa un incremento, esta vez fue de 1,93 MB/segundo, es decir más de 5 veces la velocidad alcanzada en la prueba anterior.

$$
1,93 \text { MBps } * 8=15,44 \text { Mbps }
$$

El consolidado de resultados obtenidos de los envíos se describe en la Tabla 3.

Tabla 3. Tabla de resultados de trasferencia con y sin interferencia entre los canales

\begin{tabular}{|c|c|c|}
\hline Canal usado & Promedio de Envío & Estado \\
\hline 1 & $2,71875 \mathrm{Mbps}(4)$ & Interferido \\
\hline 11 & $15,44 \mathrm{Mbps}(5)$ & No interferido \\
\hline
\end{tabular}

En las Figuras 5 y 6 se muestra una gran interferencia producida entre redes que usan simultaneamente en el canal 6, y con base en los resultados de la Tabla 3, si se ubicara una nueva red en este canal, se esperarían bajas tasas de transferencia. Estas tasas en canales que se solapan dependen del tráfico instantáneo, tal como fue posible validar en experimentos en otras pruebas realizadas en laboratorio.

\section{CONCLUSIONES}

Se realizaron pruebas experimentales que permitieron conocer cómo afecta la interferencia de canales solapados el envío de archivos en redes WiFi, y se propuso un procedimiento para realizar la instalación de una red libre de interferencias. Se demostró experimentalmente que la transmisión de archivos en canales libres de interferencia es superior a la que se realiza en canales solapados. 
Como trabajos futuros se propone cuantificar la interferencia de WiFi contra tecnologías como Zigbee, y se propone realizar el mismo estudio entre más sistemas inalámbricos utilizando el mismo canal o canales parcialmente solapados. Otras sugerencias son:

- Cambiar la selección de canal. Los Access Point que trabajan en estrecha proximidad no deben usar los canales que se superponen. Aquí, sí es posible, se debe considerar el clásico AP de panal de emplazamiento, pero es necesario tener en cuenta que en algunos equipos $802.11 \mathrm{n}$, la posición del canal secundario (Por debajo o por encima del primario) es una opción configurable por el usuario, lo que le da un grado de libertad adicional.

- En caso de que su red tenga más de un Access Point, puede que tenga que reducir la capacidad de los transmisores para mantener la interferencia a un nivel aceptable. Esta es una situación en la que mayor capacidad no significa mejor conexión. Por lo general, la capacidad óptima de transmisión de un AP es entre 5 y $10 \mathrm{dBm}$, lo que asegura una mejor densidad de los APs instalados, minimiza la carga en cada uno de ellos, y reduce al mínimo la interferencia entre los Ap vecinos, que podrían compartir el mismo canal. Eso no quiere decir que un AP poderoso es malo, pero varios APs poderosos vecinos entre sí, también se interferirán entre sí. Es por eso que se deben bajar sus capacidades.

- Otro asunto es asegurarse que los usuarios no abusen de la red con adaptadores Wi-Fi clientes de gran alcance, porque la interferencia también puede ser provocada por más de uno de estos adaptadores activos en el mismo lugar. No hay necesidad de que el adaptador cliente transmita a $50 \mathrm{~mW}$, cuando el AP está transmitiendo a sólo $5 \mathrm{~mW}$.

\section{REFERENCIAS}

[1] Guevara Calume R. (2011). Caracterización de un proceso automatizado de trabajo cooperativo con robots, para determinar la configuración topológica más confiable con nodos en movimiento en un escenario de alto tráfico e interferencia continua. Tesis de Maestría. Instituto Tecnológico Metropolitano.

[2] Kurose J.F. y Ross K.W. (2004). Redes de Computadores: Un enfoque descendente basado en internet. Pearson Addison Wesley, $740 \mathrm{p}$.

[3] Jin A.P., Seung P., Pyoung D. y Kyoung R. (2002). Analysis of spectrum channel assignment for IEEE 802.11b wireless LAN. 5th International Symposium on Wireless Personal Multimedia Communications. 
[4] Byeong G.L. y Sunghyun C. (2008). Broadband wireless access and local networks: mobile WiMax and WiFi. Artech House. $618 \mathrm{p}$.
[5] Metageek. http://www.metageek.net/ products/inssider. Online: Oct. 2012. 